\title{
Analysis and Characterization of Finite-size Curved Frequency Selective Surfaces
}

\author{
Francesco Mattiello ${ }^{1}$, Giovanni Leone ${ }^{1}$, Giuseppe Ruvio ${ }^{1,2}$ \\ ${ }^{1}$ Seconda Università di Napoli, Dipartimento di Ingegneria Industriale e dell'Informazione, Via Roma 29, 81031 Aversa \\ (CE), Italy \\ ${ }^{2}$ Dublin Institute of Technology, Antenna \& High Frequency Research Centre, Kevin Street, Dublin 8, Ireland \\ Correspondence:Francesco Mattiello, Seconda Università di Napoli, Dipartimento di Ingegneria Industriale e \\ dell'Informazione, Via Roma 29, 81031 Aversa (CE), Italy
}

Received: December8, 2014 Accepted: January 4, $2015 \quad$ Available online: February 15, 2015

doi:10.11114/set.v2i1.684

URL: http://dx.doi.org/10.11114/set.v2i1.684

\begin{abstract}
Frequency Selective Surfaces (FSSs) are spatial filters that are largely employed in radomes for radars and antennas where high performance is necessary. FSS can be categorised in terms of their frequency behaviour. Band-pass FSS structures are able to let the electromagnetic wave pass in certain frequency ranges and shield others, which are especially suitable for out-of-band stealth of radomes. The properties of frequency selectiveness of these screens are used at microwave and infrared wavelengths. By tuning the electrical size and geometry of the unit-cell (patch or aperture) different frequency behaviours of the structure are generated. Although their analysis and simulation is significantly simplified with the approximation of infinite and planar array, in some real applications FSS are required to be conformal to limited non-planar structures. The literature on the problem of characterizing finite-size and curved FSS has been sparse and more focused on the single application rather than on a unified approach. In the aim of synthesizing valuable techniques, this paper reviews significant research results on truncated and curved FSS which were presented in the dedicated literature.
\end{abstract}

Keywords: microwave engineering, frequency selectiveness, RADAR, periodic structures, floquet's theory, method of moments, asymptotic boundary conditions.

\section{Introduction}

Frequency selective surfaces (FSS) have been studied for the last five decades and comprehensive books have been also published (Munk, 2000) and (Wu, 1995). FSS have evolved from simple canonical forms to the complex geometries known today. This evolution has been driven primarily by increasingly stringent performance requirements of recent applications. The progress of frequency selective surfaces was also motivated by the significant improvements in the methods of analysis, from the computing capacity and on the manufacturing technology. Moreover, being FSS part of the broad family of artificial materials (Ruvio\& Leone, 2014), new research efforts have been dedicated to this topic.

The frequency selective surfaces can be fabricated as planar two-dimensional periodic arrays of metallic elements with special geometric shapes, or may be created by periodic openings in a metal screen. The transmission and reflection properties of these surfaces are dependent on the operating frequency and may also depend on the polarization and the angle of the electromagnetic plane wave impinging the material (angle of incidence). These structures are used when it is required that in some frequency band a surface is fully opaque (stop-band) and in other bands the same surface allows the transmission of the electromagnetic wave. This behaviour can be very advantageous, for instance, in space applications for a telescope or satellite in orbit.

In passive planar FSS the power is supplied from a plane wave that affects the structure, while in the active ones each element has its own power source. In the passive array case with elementary dipoles, the incident wave will be partly transmitted in the forward direction $\left(E_{t}\right)$ and partly reflected in the specular direction $\left(E_{r}\right)$. Under resonant condition and without grating globes, the amplitude of the transmitted wave $\left(E_{t}\right)$ is minimum and the reflected wave is $\left(E_{r}\right)$. If we define a reflection coefficient $\left(\Gamma=E_{r} / E_{t}\right)$, we have the maximum value of this coefficient, for a specific frequency. It is possible to make a complementary structure, through openings in a metal plane. In this case, we have the maximum value of the transmission coefficient, for a specific frequency. Therefore for an array of dipoles the behaviour of the 
structure is similar to a reject-band filter, while for an array of slots it is similar to a pass-band filter (Figure 1).

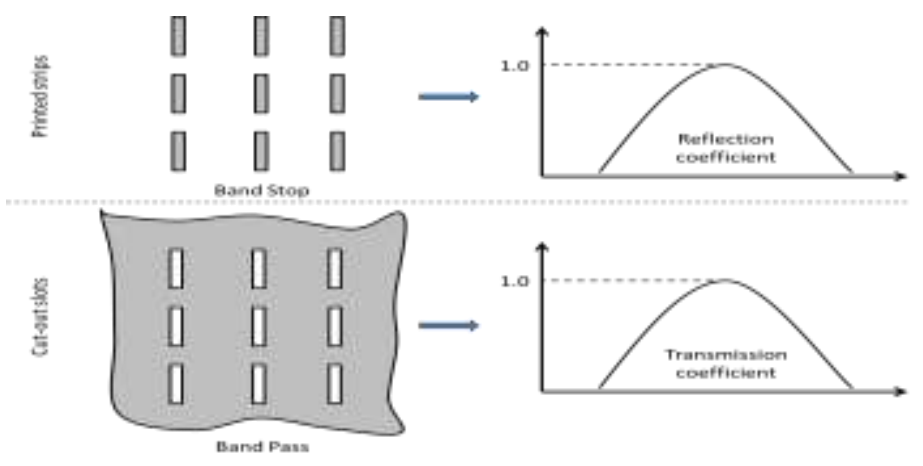

Figure 1.Basic operating principles of strip- and slot-based FSSs

To understand the physical principle underlying the FSS, it is possible to use a circuit analogy, through the use of LC-elements. For example, if we consider a row of two-dimensional array of dipoles, each element of this structure can be shown schematically by means of a series of dipoles $\mathrm{LC}$, in which the inductance $\mathrm{L}$ is the self-inductance of each dipole, and the capacity $\mathrm{C}$ represents the gap between an element and the next. By suitably dimensioning the individual elements is possible to obtain that the reactive part of this series LC vanishes and the impedance of the dipole will be the minimum. If this condition is verified and the FSS structure is hit by an electromagnetic wave, a maximum possible interaction is obtained.

In order to achieve a filtering behavior which is independent on the angle of incidence of the wave and its polarization it is necessary to create structures that have more complex geometries than dipoles and slots. Shapes as crosses, "y" and rings are used. Moreover, to improve the frequency selectivity, layered structures are often used.

\section{Recent Research Trends}

Typical FSS applications are also desired in the design of hybrid radomes shielding antennas from external weather conditions. They can also lower the Radar Cross Section (RCS), outside of the frequencies of interest. If we consider, for example, an antenna mounted on the nose of an airplane, one FSS disposed on the surface of the radome allows to scatter the incident radiation that operates outside the band of interest in the bi-static direction, with the consequent result of a return very low in the direction of backscatter. A similar FSS application is in the design of band-stop filters. An example of this application is related to the realization of a hybrid radome applied on a shipboard radar antenna.

A recent solution to reduce the Radar Cross Section of a Microstrip Antenna using an FSS is presented in (Genovesi, Costa, \& Monorchio, 2012). The goal is accomplished by replacing the solid ground plane of the device with a hybrid structure comprising a suitable FSS. For this purpose a microstrip line with characteristic impedance equal to $50 \mathrm{Ohm}$ has been printed on the top of an FR4 dielectric slab. On the opposite side of the FR4 slab, three different grounds have been printed: (a) a solid ground plane, (b) an FSS ground, and (c) a hybrid ground comprising a PEC area beneath the microstrip line surrounded by FSS unit cells. The simulated and measured results has shown that the antenna with the hybrid ground planeis characterized by a strong RCS reduction within the transmission band of the FSS since the incoming signals passes unaltered through the multilayer. The RCS reduction in the same frequency band is preserved also for oblique incident angles since the frequency response of the FSS is characterized by a high angular stability. The proposed design does not affect the in-band performance of the radiating device allowing, at the same time, to decrease the out-of-band radar signature of the target. Therefore, it represents the best solution among the investigated ones. The joint study of the response of the FSS for plane waves and for quasi-TEM waves has revealed a suitable and efficient approach to provide a fast design of a low-RCS antenna, avoiding time-consuming full-wave simulations. Measurements on a real prototype con firm the validity of the proposed design.

FSSs find also application in the integration of circuit analog absorbers. These genarally consist of crossed elements made of a resistive material backed by a ground plane. These surfaces are similiar to the classical Salisbury screen, but they are superior to them. In fact periodic surfaces exhibit a reactive component that, to a large extent, can be designed to produce a larger band with particularly when more layers added. Periodic meanderlines can be used for this purpose (Figure 2). If the structure is hit by an incident wave with an E-field aligned with the meanderline, the structure will act like a shunt inductance over an equivalent transmission line; whereas an E-field perpendicular with the meanderline will see a capacitive load. If an incident wave is linearly polarized and tilted $45^{\circ}$, it may be decomposed into vertical and horizontal components where the former is delayed and the latter advanced. If after passing through the polarizer, the net difference between the two components is $90^{\circ}$, they will recombine into a circular polarized wave. Cascading two or 
more polarizer sheets can improve the bandwidth and adding dielectric slabs can improve the angle of incidence stability.The PEC ground plane can be replaced by a high-impedance surface (HIS),then a phase delay greater than 180 degrees can be obtained and the physical thickness can be reduced (Seman, Cahill, \& Fusco, 2009).The absorbing bandwidth of the Salisbury screen can be increased by employing more resistive sheets spaced approximately quarter wavelength apart, often referred to as a Jaumann absorber (Chambers\& Tennant, 1994) or by placing dielectric slabs above and below the resistive sheets (Munk, B.A., Munk, P. \& Pryor, 2007).These structures are, however, unsuitable for deployment in applications where the major design driver is to reduce physical thickness. Recently, it was shown that an analysis of the absorbing structure by a simple equivalent circuit that allowed a detailed explanation of the key parameters for the design of the absorber. The authors quantified the relationship between the surface resistance of the ink-film composing the screen and the reflectivity bandwidth (Costa, Monorchio, \& Manara, 2010).The work described in this paper was motivated by the need to widen the absorption band of the Salisbury screen absorber. The resistive Salisbury screen was replaced by a high-impedance surfaces (HIS) comprising lossy frequency selective surfaces (FSS) such as a periodic array of conductive patch elements. The structure under analysis was described by means of an equivalent circuit approach.

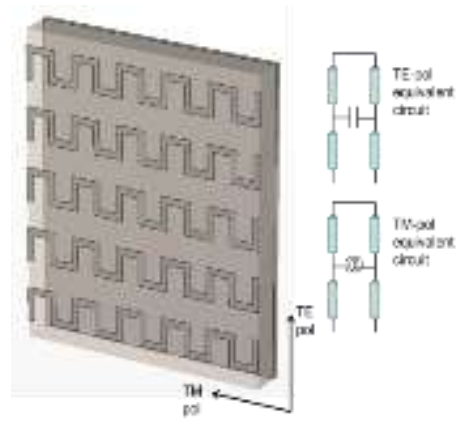

Figure 2.FSS-based absorber

Improved manufacturing techniques and the exploration of higher regions of the spectrum have facilitated more sophisticated FSS designs. Multifractal geometries were proposed for the integration of dual/wide band FSS for applications in S- and X-band (Braz \& Campos, 2014). By combining Koch curve with Sierpinski Carpet, resonance and bandwidth can be easily controlled. Good broadband behavior in combination with a high degree of miniaturization was achieved by interleaving bundles of small square patches (Semyoung, Hanjun, Joo-Ho \& Gil-Young, 2014). With the research advancements in new materials and manufacturing techniques FSSs for the terahertz spectrum have been recently proposed. At $\mathrm{THz}$ frequencies, the thicknesses of commercially available substrates are comparable to the free-space wavelength. As a result, the substrate plays a critical role in determining the transmission characteristics of THz multilayer FSS structures. Proper coupling method between FSS structures should be chosen to avoid unwanted substrate resonances or Fabry-Pérot resonances, which otherwise degrade the transmission characteristics of the cascaded FSS structure. A cascaded structure was proposed to avoid multiple reflections within the substrate (Vegesna, Zhu, Bernussi, \&Saed, 2012). The transmission response is improved by introducing an extra transmission zero at a frequency location lower than the resonant frequency, thereby achieving high roll-off rate for the lower side of the stop band, and to suppress unwanted resonances, thereby increasing the rejection bandwidth of the filter. The electric properties of vanadium dioxide $\left(\mathrm{VO}_{2}\right)$ and sapphire substrates have been successfully employed for the manufacturing of FSS structures for the THz spectrum (Zhao, Lee, Zhu, Nazari, Chen, Wang, Bernussi, Holtz, \& Fan, 2012) and (Chen, Zhu, Zhao, Lee, Wang, Bernussi, Holtz\& Fan, 2010). An innovative reconfigurable THz FSS polarizer was integrated by combining $\mathrm{VO}_{2}$ patches with metallic resonators (Vegesnaa, Zhuab, Zhaoab, Fanab, Bernussiab \& Saeda, 2014). Vanadium dioxide behaves as an insulator at room temperatures and as a metal at high temperatures with a characteristic insulator-metal transition temperature of $\sim 68^{\circ} \mathrm{C} . \mathrm{VO}_{2}$ properties were also used to realize a reconfigurable single- to dual-polarized bandpass FSS structure at $0.5 \mathrm{THz}$.Other innovative nano-structure that found application in the design of FSS structures which operate in the THz spectrum are the single-walled carbon nanotubes (SWCNTs) (Ren, Pint, Arikawa, Takeya, Kawayama, Tonouchi, Hauge, \& Kono, 2012). The authors presented a terahertz polarizer built with stacks of aligned exhibiting ideal broadband terahertz properties: $99.9 \%$ degree of polarization and extinction ratios of 10-3 (or $30 \mathrm{~dB}$ ) from $\sim 0.4$ to $2.2 \mathrm{THz}$. Compared to structurally tuned and fragile wire-grid systems, the performance in these polarizers is driven by the inherent anistropic absorption of SWCNTs that enables a physically robust structure. Supported by a scalable dry contact-transfer approach, these SWCNT-based polarizers are ideal for emerging terahertz applications.

One of the challenging problems encountered while designing FSSs is to simultaneously achieve a wide bandwidth, as well as stability with respect to waves with different polarizations and angles of incidence. In the recent past, several 
techniques have been applied to control the bandwidths of FSSs, which includes choosing certain element shapes and three-dimensional layouts to provide large angular stability (Hu, Zhou, Wu, Zhou, \& Yin, 2009) and (Yang, Gong, Zhang, Zha \& Ling, 2009). In (Wu, Zhang, Xu, Yang \& Lin, 2014) a 3-D FSS structure made of honey-combed spiral metallizations shows very good stability across the operating bands for WiFi, Blue Tooth and WLAN communications. High degree of miniaturization in combination with good angle stability is reported in ( $\mathrm{Li}$, Zhang, Yang, \&Wu, 2012). Its first layer is inductive to generate band-pass behavior, while the second layer is capacitive-designed so that the miniaturization characteristic can be further improved. Compared with the traditional single-layer structure, the profile of the FSS proposed is relatively small with the cell's dimension of only $0.0814 \lambda \times 0.0814 \lambda$.

More recently all-dielectric FSS structures have been also proposed in the literature (Yu, Qu, Xu, \& Wang, 2011). In (Yu, $\mathrm{Qu}$, Wang, Huang \& Wang, 2014) it is presented the design of FSS made entirely by dielectric materials. High-permittivity materials have plasma liquid property as the metal and this property is used to design FSS with good band-pass characteristics.

\section{Grating Lobes}

An issue related to the FSS is the undesired generation of grating globes, the spatial replication of the main lobe. If we consider one dimensional periodic structure with inter-element spacing $D_{x}$ and with an incident plane wave at an angle of incidence $\eta$, it is clear that each element will be delayed in phase by $\beta D_{x} \sin \eta$ with respect to its neighbor. However in the forward as well as the specular directions, the same element will be ahead in phase or we will always obtain plane waves that can propagate in these directions (Figure 3a). There are possibily other directions where propagation can take place as illustrated in Figure 3b.

Here the incident wave is the same as before and a possible grating globe direction is denoted by $\eta_{g}$. From Figure $3 \mathrm{~b}$ we see that the total phase delay of an elementis going to be $\beta D x\left(\sin \eta+\sin \eta_{g}\right)$, where $\beta=2 \pi / \lambda_{g}$ and $D_{x}$ is the interelement spacing. If this delay is equal or a multiple of $2 \pi$, all signals from the elements will be in phase in the direction $\eta_{g}$, satisfying the following condition:

$$
\beta D x\left(\sin \eta+\sin \eta_{g}\right)=2 \pi n
$$

In other words, if the phase delay of the two collinear points, which distance is $D x$, is $2 \pi n$ in the $\eta_{g}$ direction a grating lobe will occur with a consequent degradation of the FSS performance. Since grating lobes are only a function of frequency and element spacing, it is not possible to avoid grating lobes at all frequencies. It is mostly important to be aware of their presence at the edge of the operating bandwidth in the design of the FSS structure.

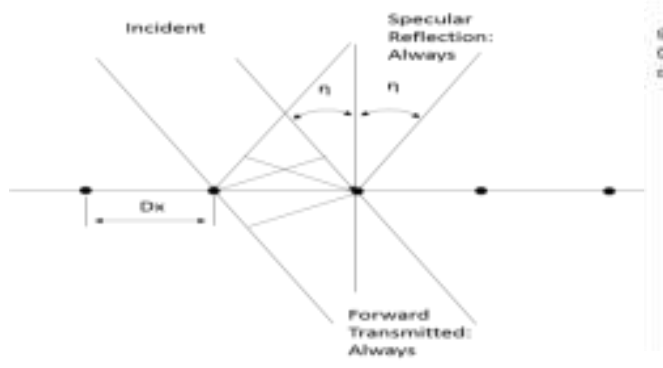

(a)

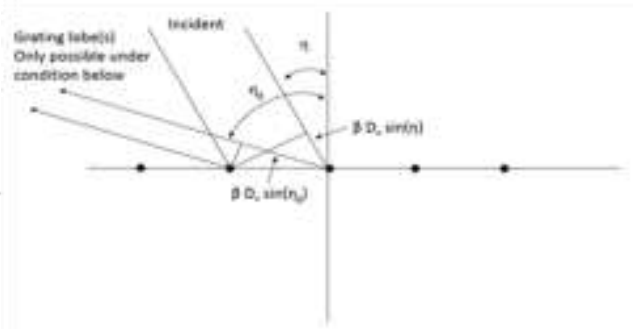

(b)

Figure 3. Grating lobes condition

\section{Frequency Selectiveness}

When designing a band-pass filter and band-stop FSS the choice of the cell-unit is crucial. Some elements are inherently more broad banded or more narrow-banded than others. Also the bandwidth of an FSS can in general be varied significantly by variation of the inter-element spacings $D_{x}$ and $D_{z}$ : a larger spacing will in general produce a narrower bandwidth and vice versa. Howewer two aspects must be observed closely: the grating globes and the variation of the first resonant frequency with angle of incidence and polarization. The elements are arranged into four categories:

1. Center connected or N-poles, such as the simple straight element, three-legged element; anchor elements; the Jerusalem cross and the square spiral.

2. The loop types such as the three- and four-legged loaded elements; the circular loops; and the square and hexagonal loops.

3. Patch types of various shapes.

4. Combinations of the previous categories.

The simplest geometry of the first group to analyse is the simple straight element, so-called Gangbuster surface first 
presented in (Kornbau, 1984). It consists of these elements arranged into a bidimensional array, which presents the maximum interaction with an incident plane wave when the length of the elements is approximately $\lambda / 2$. The interelement spacings $D_{x}$ and $D_{z}$ can modify the bandwidth response, showing a broadband response if $D_{x}$ and $D_{z}$ are reduced and the resonant frequency remains essentially constant while the onset of grating globes is delayed. The drawback of this simple geometry is the dependancy from the polarization, in fact this structure interacts only with polarization parallel to the structure itself. Obviously it is possible to handle an arbitrary polarization using two of these surfaces twisted $90^{\circ}$ with respect to one other.

Another geometry of the first group is the unloaded tripole array, which have the advantage of a larger bandwidth. Another advantage respect to the straight element is the lower cross polarization, although it is more complicated to be manufactured. The combination of complex unit-cells leads to so-called super-dense surfaces, which are discussed in detail in (Schneider, 1992). It is possible to modify this structure, adding an end capacity, resulting into the anchor element. It naturally leads to smaller elements resulting in significantly smaller interelement spacing.

Into the second category we find the loop types, which can have different shapes, for example the three legged and the four legged loaded element. They have the advantage to control bandwidth by modification of the load impedance $Z_{L}=j 1 / 2 Z_{0} \tan (\beta l)$, related to the length $l$ of the leg of the element. However, the major advantage of the three legged elements is into the better displacement on the surface, resulting into considerably smaller inter-element spacings $D_{x}, D_{z}$. Into the third group we find the solid interior elements of simple shapes such as squares, hexagons and circular disks. If we consider a periodic surface of square apertures, this class of surfaces leads to element dimensions close to $\lambda / 2$, resulting into a larger inter-element spacing leading to angle of incidence sensitivity and early onset of grating globes.

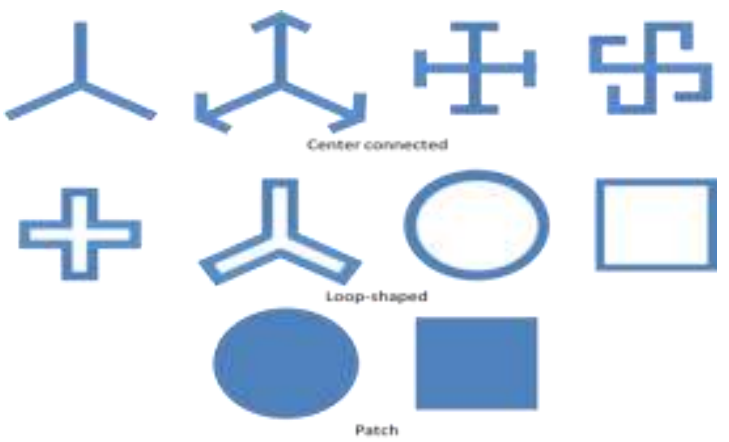

Figure 4. Examples of FSS unit-cells

\section{Modelling Period Structures}

There are basically two ways to determine the scattering and impedance properties of periodic structures:

1. The mutual impedance approach, sometimes called element to element approach, which is closely associated with the Method of Moments (MoM).

2. The plane wave expansion or the spectral approach.

Let us focus our attention to the first method. If we consider a finite array structure, this is not a periodic structure any longer. However, it may be a realistic approximation of a periodic structure provided that all inter-element spacings are the same, $D_{x}$ as well as $D_{z}$. Let us now assume that this array is actively fed by voltage generators $\mathrm{V}_{1} \ldots \mathrm{V}_{9}$ as shown in Figure 5.The current $I_{2}$ in antenna 2 will produce a field at antenna 1 which in turn results in an impressed voltage defined as $-Z_{1,2} I_{2}$ where $Z_{1,2}$ is the element mutual impedance between antennas 1 and 2 . If antenna 1 also has a generator impressing the voltage $V_{1}$ at its terminals, then the total impressed voltage will be $\left(V_{1}-Z_{1,2} I_{2}\right)$. According to Ohm's law, this total impressed voltage must be equal to the self-impedance $Z_{1,1}$ plus the load impedance $Z_{L 1}$, multiplied by the current $\mathrm{I}_{1}$ :

$$
V 1=\left(Z_{1,1}+Z_{L 1}\right) * I_{1}+Z_{1,2} * I_{2}
$$

We can rewrite the previous expression for the multi-element case:

$$
\begin{gathered}
V_{1}=\left(Z_{1,1}+Z_{L 1}\right) * I_{1}+Z_{1,2} * I_{2}+Z_{1,3} * I_{3}+\cdots+Z_{1,9} * I_{9} \\
V_{2}=Z_{2,1} * I_{1}+\left(Z_{2,2}+Z_{L 2}\right) * I_{2}+Z_{2,3} * I_{3}+\cdots+Z_{2,9} * I_{9} \\
\vdots \\
V_{9}=Z_{9,1} * I_{1}+Z_{9,2} * I_{2}+Z_{9,2} * I_{2}+\cdots+\left(Z_{9,9}+Z_{L 9}\right) * I_{9}
\end{gathered}
$$

We can notice that the mutual impedance terms into the previous expression are not the same as for the two-element 
case, because the shape of the current distribution $I(z)$ may change due to the presence of the other elements. All the mutual impedance terms are time-consuming to evaluate, because they are obtained by a numerical integration. If the structure under investigation is very large, the impedance matrix will soon grow to be a computationally heavy size.

Let us now extend the previous formulation to a single infinite case, where the array is infinite into the vertical direction (z-axis). We will require the inter-element spacings in the z-direction to be the same $\left(D_{z}\right)$ for all the columns; however, the spacings in the horizontal plane along the $\mathrm{x}$-axis need not be uniform.

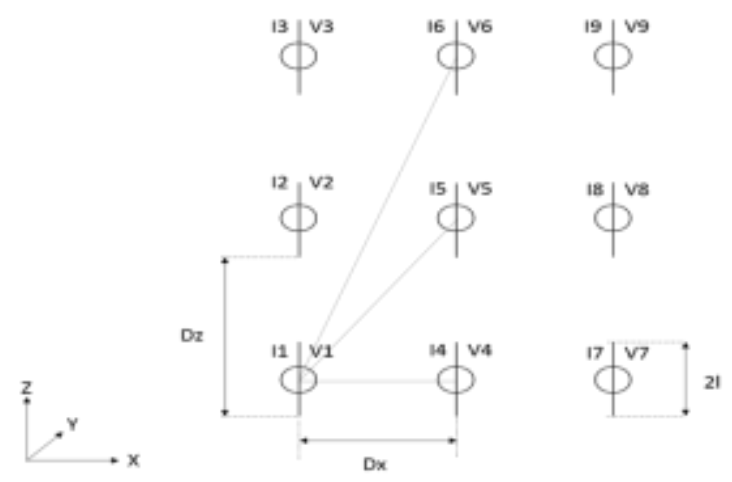

Figure 5. Array of radiating elements

If this structure is exposed to an incident plane wave propagating in the $\hat{s}$ direction:

$$
\hat{s}=\hat{x} s_{x}+\hat{y} s_{y}+\hat{z} s_{z}
$$

it will induce the currents $I^{(I)}, I^{(2)}, \ldots, I^{(Q)}$ in the arbitrarily chosen reference elements of columns $I, 2, \ldots Q$, respectively. Furthermore the element currents in a particular column will have the same amplitude as that of its reference element and a phase matching the phase velocity of the incident signal in the z-direction

$$
I_{q m}=I_{q} e^{-j \beta m D_{z} s_{z}}, \quad q=1 \ldots .,
$$

Where $m$ is equal to the element number in the z-direction with $m=0$ corresponding to the reference element in the respective column. With this equation, we can simplify the previous equation of the multi-element case as:

$$
\begin{gathered}
V^{(1)}=\left(Z^{1,1}+Z_{L 1}\right) * I^{(1)}+Z^{1,2} * I^{(2)}+\cdots+Z^{1, Q} * I^{(Q)} \\
V^{(2)}=Z^{2,1} * I^{(1)}+\left(Z^{2,2}+Z_{L 2}\right) * I^{(2)}+\cdots+Z^{2, Q} * I^{(Q)} \\
\vdots \\
V^{(Q)}=Z^{Q, 1} * I^{(1)}+Z^{Q, 2} * I^{(2)}+\cdots+\left(Z^{Q, Q}+Z_{L Q}\right) * I^{(Q)}
\end{gathered}
$$

where the term

$$
Z^{q, q^{\prime}}=\sum_{m=-\infty}^{\infty} Z_{q, q^{\prime} m} e^{-j \beta m D_{Z} s_{Z}}
$$

is called the array mutual impedance. This term is made of a sum of element mutual impedances $Z_{q, q^{\prime} m}$. Although this formulation leads to some reduction in computational time, it should be emphasized that the evaluation of the array mutual impedances $Z^{q, q^{\prime}}$ based on equation (7) is still time-consuming. This occurs simply because they rely on evaluating element mutual impedances. In addition, the infinite series (7) is in general very slowly convergent and requires the incorporation of many elements, namely high values of $m$. But there is an alternative approach based on the evaluation of the array mutual impedance between two columns with co-linear elements located in a cylindrical coordinate system using another infinite series with Hankel functions of second kind of order 0 .

This single infinite scenario can be easily extended to a double infinite case, giving an infinite $\times$ infinite periodic structure, as long as we keep the inter-element spacings $D_{x}$ and $D_{z}$ constant. If this configuration is exposed to an incident plane wave propagating in the direction $\hat{s}$, then the amplitude of all the element currents will be the same, while their phases will match the phase of the incident field. Formally, for the element current in column $q$ and row $m$ we have:

$$
I_{q m}=I_{q} e^{-j \beta m D_{x} s_{x}} e^{-j \beta m D_{z} s_{z}}
$$


As in the single infinite case above, this is a direct consequence of Floquet's theorem (Floquet, 1883).

\section{Curvature and Truncation Effects}

Frequency Selective Surfaces (FSSs) are spatial filters that are largely employed in radomes for radars and antennas where high performance is necessary. FSS can be categorised in terms of their behaviour in frequency. Band-pass FSS structures are able to let the electromagnetic wave pass in certain frequency ranges and shield others, which are especially suitable for out-of-band stealth of radomes. The properties of frequency selectiveness of these screens are used at both microwave and infrared wavelengths for the design of components in reflector antenna systems (O'Nians \& Matson, 1966) as well as components in optical systems (Ulrich, 1967), respectively. FSS screens are practically made of arrays of conducting patches, or arrays of apertures within a conducting flat or curved surface. By tuning the electrical size and geometry of the unit-cell (patch or aperture) different frequency behaviours of the structure are obtained. However, more diverse spectral response may be achieved by using multiple layers of the arrays and array substrates combined into multi-layered structures.

The FSS integrated for many practical applications are electrically large - on the order of 100 wavelengths per side. Due to the necessity of large size, planar FSS may be ideally modelled as infinite planar periodic arrays of passive elements. When illuminated by a plane wave, currents are induced and scattered fields are generated with a periodicity equal to that of the FSS structure. The occurrence of this periodicity allows the Floquet's modelling of the scattering problem. As the response of the full structure can be expressed in terms of the single periodic cell, the analysis is significantly reduced. Large FSS screens that would imply to an excessive computational burden are therefore remodelled by infinite arrays which are compatible with the Floquet's condition and easy to study. The reflection and transmission coefficients of planar and electrically large screens present good agreement between measurements and analytic prediction based on the infinite model formulation.

However for smaller or curved FSS screens effects due to an alteration of the periodicity cannot be disregardable and the infinite planar model results may strongly disagree from measurements on the physical structure. This situation is the most common in reality when the FSS is used as a radome or as a parabolic sub-reflector in large antenna systems. Non-zero curvature and finite size of the FSS screen alter the periodicity of the unit-cells with a consequent divergence from Floquet-based anaylsis. Moreover, alteration of periodicity precludes the possibility of the analysis based on the characterization of a single periodic cell with dramatic consequences on the computational burden. For instance, to quantify the computational efforts required of surface sizes on the order of ten to 100 wavelengths on a side, the number of elements would be on the order of 100 to 10000 (assuming one element per square wavelength). As the size of each unit-element is still on the order of a wavelength, any full-model formulation would require a number of unknown basis functions (perhaps five to 50 depending on the geometry) to approximate the current on a unit-cell, with the overall number of functions becoming quite large. Hence, an accurate analysis and solution of the fields scattered becomes overbearing, if not impossible.

Although FSS structures have been widely documented in the last 60 years, dedicated literature on either planar or curved truncated periodic surfaces has been relatively scarce and sparse. Kastner and Mittra (Kastner \& Mittra, 1984) introduced iterative solutions to scattering equations modelling the finite screen with arbitrarily shaped bodies. In particular, the spectral domain approach was combined with the conjugate gradient (CG) method to derive the solution of a finite-sized FSS screen that requires a large number of unknowns for its numerical modelling. Because the number of basis functions used in the analysis is limited to what can be practically computed, the anaylsis may be considered approximate for surfaces generally used in practice. Curved surfaces have been extensively used in practice (Dick, 1981), (Agrawal \& Imbriale, 1979), although limited attempts have been made to analyze the effects of curvature with a rigorous approach.

Cwik and Mittra (Cwik \& Mittra, 1988) investigated the curved strip grating of one-dimensional truncation and proposed rigorous numerical solutions as well as local planar approximations. Therefore, the combined effects of truncation and curvature of periodic surfaces were examined. Initially, equations were outlined for surfaces which are finite and curved in two dimensions (presenting a local periodicity), as well as infinitely periodic in one dimension but truncated and curved in the second. By removing this periodicity, a truncated strip grating results and the scattered fields as well as an associated reflection coefficient were calculated. These numerically rigorous calculations were compared against two approximate solutions. The comparison is meant as a check of the approximate solutions with an eye toward using them in the analysis of surfaces which are finite and curved in two dimensions.

Although the calculation of finite curved surface of arbitrary elements is not both accurate and fast so far, several attempts have been made. Philips et al. have put forward two methods based on Pocklington's equation (Philips, Parker \& Langley, 1995). They applied this method on finite arrays of dipoles arranged on a cylindrical surface with different radii of curvature and illuminated by an incident (TM) plane wave field arbitrary to extract frequency selective characteristics and evaluate the effect of mutual coupling on the transmission characteristics of the arrays. The same 
authors also proposed a technique based on ray tracing to investigate an FSS surface embedded in a curved dielectric. The FSS was treated as an infinitesimally thin interface with known complex reflection and transmission coefficients subjected to multiple interactions with rays internally reflected within the dielectric.The transmission properties of such arrays were studied as the curvature increases for the case of paraboloids illuminated by plane waves, and also when enclosing a finite aperture-type antenna.

In recent years, some alternative methods using hybrid volume-surface integral equation were presented. A hybrid volume-surface integral-equation approach to analyze the transmission and reflection characteristics of finite and curved FFS structures was introduced ( $\mathrm{Yu} \& \mathrm{Lu}, 2005$ ). The hybrid integral equations were obtained using the surface- and volume-equivalent principles with two advantages: the capability of modelling arbitrarily shaped FSS structures in detail; the multilevel fast multiple algorithm to speed up the solution process. From the analysis of the scattering characteristics and frequency responses of several finite-sized FSSs, important effects due to the non-zero curvature were characterized. In particular, it was shown that by reducing the radius of curvature, amplitude variation, frequency shift and bandwidth change in the reflection and transmission responses occurred.

Stupfel and Pion considered the time-harmonic electromagnetic scattering problem from a finite planar or curved infinitesimally thin FSSs which are made of unit-cells simplified exclusively by electric conductors and free-space (Stupfel \& Pion, 2005). In order to avoid the meshing of these cells and accelerate processing, the problem was addressed in terms of an integral equation formulation in conjunction with approximate impedance boundary conditions (IBC) which are assigned to the sheet that models the FSS. The impedance in the IBC model is originally derived from the exact reflection coefficient calculated, for the fundamental Floquet mode, on the infinite planar FSS illuminated by a plane-wave with a certain incidence. When the curvature of the FSS diverges from zero and/or the direction of the incident wave is unknown, higher order IBCs were proposed. These modes remain valid for a large angular range and can be implemented in standard MoM formulation. Finally, the impedance is approximated by its truncated Fourier series that considerably enhances the accuracy of the results although at the cost of a denser mesh.

A useful investigation on non-planar arrays was carried out by Šipus et al. (Šipus, Lanne \& Josefsson, 2006) and (Šipus, Burum, Skokic \& Kildal, 2006). Although selectiveness in frequency was not the targeted application, the analysis of circular cylindrical arrays of waveguide elements and of spherical arrays of microstrip antennas introduces suitable techniques for the investigation of curved FSSs structures. The analysis method is based on the modal solution, hence on the superposition of one-dimensional spectral solutions and on the MoM. The modal approach requires the summation of a large number of terms in the Fourier series or vector-Legendre transformations for structures with large radii and the occurrence of highly oscillating integrals. Asymptotic formulas are used for calculating the Green's functions with a suitable definition of the integration contour for calculating the inverse transformation into the spatial domain.

Bosiljevacand Šipus also presented a hybrid approach for the analysis of different curved antenna arrays or reflecting/transmitting surfaces that finds immediate application for the analysis of FSSs structures (Bosiljevac \& Šipus, 2008). The approach is based on a rigorous spectral domain technique and a MoM which are then combined with an appropriate asymptotic method in order to accelerate the computational duration of the problem. Merging of the two methods is achieved by applying asymptotic extraction in the calculation of the reaction integrals of the MoM matrix. This combination offers the accuracy of the spectral domain method and the speed of the asymptotic approach.

Meng et al. proposed a numerical method which is based on the combination of the mutual admittance approach as well as the spectral domain method of finite planar FSSs to solve curved slot arrays problems (Meng, Lu, Wu \& Huang, 2009). This method is employed to study the effects of non-zero curvature curved effects on one-dimensional truncated slot arrays by focusing on the magnetic current distribution, scattering patterns and transmission characteristics.

The solutions of finite curved FSSs are all with respect to local planar approximation method, which are limited and only in some certain ranges could the obtained results agree well with that of experiments. On the other side, most studies are mainly involved in the calculation methods of curved FSS and transmission characteristics. Few analyses are carried out to study the mechanism of curved effects on FSS arrays.

\section{Numerical Methodologies}

The study of FSS can be essentially reduced to a problem of scattering by a conductor body, and for this purpose, for several years the MoM technique - also known as a method of weighted residuals (Harrington, 1993) -has been used. This method is typically used as the reference against which the new frequency-domain integral equation approaches are compared in terms of accuracy, CPU-time and memory. In the conventional implementation of the MoM, a typical sampling rate of $\lambda / 10$ is employed for the discretization, along with the subdomain basis functions, such as Rao-Wilton-Glisson (RWG) (Rao, Wilton \& Glisson, 1982) or rooftops (Glisson \& Wilton, 1980), used to represent the induced currents. The total number of sub-sectional basis functions employed to cover the target surface determines the size of the system matrix that is needed to solve for multiple right-hand sides, each of them corresponding to a different excitation. However, due to a fine discretization 
of the object geometry (typically $10 \times \lambda$ in the conventional MoM), the matrix size becomes large relatively quickly as the object size becomes electrically large, and this, in turn, places a heavy burden on the CPU, both in terms of solving time and memory.

In the past, the use of asymptotic high-frequency techniques (Kouyoumjian, 1965; Knott, 1985) was the only viable option for addressing such large problems. However, several techniques have recently been developed to ease the computational burden imposed on the conventional MoM. One of these techniques is based on a strategy that utilizes a domain-decomposition scheme and reduces the number of unknowns by replacing the subdomain-type basis functions with a set of macro-basis functions. Some significant works that fall in this category can be found in (Li \& Chew, 2007; Kron, 1953; van de Water, de Hon, van Beurden, Tijhuis \& de Maagt, 2005; Laza, Matekovits \& Vecchi, 2005; Matekovits, Vecchi, Dassano \& Orefice, 2001).

The Characteristic Basis Function Method (CBFM) (Prakash \& Mittra, 2003; Pelletti, Bianconi, Mittra, \& Monorchio, 2013; Pelletti, Bianconi, Mittra \& Monorchio, 2013b) works with a set of high-level basis functions, called CBFs, which are defined over relatively large domains, though they are expressed in terms of the conventional subdomain functions. The CBFs are tailored to take into account the geometrical properties of the surface upon which they are defined; hence, these bases are not limited to a predetermined and/or fixed shape, e.g., planar facets. The underlying objective of the CBFM is the reduction of the number of unknowns that arise from the discretization process when applying the conventional MoM. Their use leads to manageable-sized matrices, called reduced matrices, which are amenable to direct solution in many cases, without relying upon iteration or preconditioning. An important consequence of this is that multiple right-hand sides can be handled efficiently. Furthermore, for very large problems, the reduction achieved in the number of unknowns translates into significant CPU-time saving if an iterative solution process is utilized afterwards to solve the reduced matrix.

Table 1.Summary of notable numerical techniques for FSS simulation

\begin{tabular}{|c|c|c|c|}
\hline Numerical technique & Description & Pros & Cons \\
\hline Finite Element Model & $\begin{array}{l}\text { Most general, handles complex } \\
\text { material and geometries, volume } \\
\text { mesh and field equation, } \\
\text { numerically exact solution }\end{array}$ & $\begin{array}{l}\text { Can handle complex geometries } \\
\text { and inhomogeneus medium }\end{array}$ & $\begin{array}{l}\text { It is necessary to discretize } \\
\text { (mesh) the entire volume under } \\
\text { investigation }\end{array}$ \\
\hline $\begin{array}{c}\text { Integral Equation (Method of } \\
\text { Moments) }\end{array}$ & $\begin{array}{l}\text { Efficient solution for open } \\
\text { radiation and scattering } \\
\text { problems, currents solved on } \\
\text { surface mesh, efficient with } \\
\text { structures are primarily metallic }\end{array}$ & $\begin{array}{l}\text { It is necessary to discretize only } \\
\text { the regions where the metal } \\
\text { objects are present. Good for } \\
\text { electrically large objects. }\end{array}$ & $\begin{array}{l}\text { Not possible to simulate } \\
\text { complex geometries and } \\
\text { inhomogeneus medium and } \\
\text { broadband analysis }\end{array}$ \\
\hline $\begin{array}{c}\text { MoM with Charateristic Basis } \\
\text { Function }\end{array}$ & $\begin{array}{l}\text { Evaluation of the independent } \\
\text { Charateristics Basis Functions } \\
\text { using an SVD operation on the } \\
\text { evaluated currents induced by a } \\
\text { few incident plane waves. }\end{array}$ & $\begin{array}{l}\text { Reduced size of MoM Matrix; } \\
\text { this results in a strong } \\
\text { compression of the MoM matrix, } \\
\text { which allows an iteration-free } \\
\text { (e.g., LU decomposition) } \\
\text { solution also for electrically } \\
\text { large problems }\end{array}$ & $\begin{array}{l}\text { It is necessary a preliminary step } \\
\text { to calculate the orthogonal } \\
\text { CBFs, calculated through an } \\
\text { SVD operation, that increases its } \\
\text { computational burden if the } \\
\text { electrical size of the blocks } \\
\text { increases }\end{array}$ \\
\hline $\begin{array}{l}\text { Equivalent LC circuit for } \\
\text { multi-layered non resonant FSS } \\
\text { (Monorchio\&Manara 2012) }\end{array}$ & $\begin{array}{l}\text { Evaluation of the equivalent LC } \\
\text { parameters, for using the } \\
\text { generalized transmission line } \\
\text { model }\end{array}$ & $\begin{array}{l}\text { Can be employed for a } \\
\text { preliminary } \\
\text { and fast analysis of } \\
\text { frequency-selective surfaces } \\
\text { after a trivial } \\
\text { pre-processing to determine the } \\
\text { lumped parameters. It is non } \\
\text { necessary to do a 3D Full } \\
\text { analysis to predict the frequency } \\
\text { response of the structure }\end{array}$ & $\begin{array}{l}\text { It would } \\
\text { be of particular usefulness in the } \\
\text { design of complex structures, } \\
\text { where the frequency-selective } \\
\text { surface is only one of the } \\
\text { building } \\
\text { blocks }\end{array}$ \\
\hline $\begin{array}{l}\text { Equivalent LC circuit for } \\
\text { multi-layered non resonant FSS } \\
\text { (Jayaprakash\&Kavitha 2014) }\end{array}$ & $\begin{array}{l}\text { Evaluation of the equivalent LC } \\
\text { parameters, for using the } \\
\text { generalized transmission line } \\
\text { model }\end{array}$ & $\begin{array}{l}\text { It is non necessary to do a } 3 \mathrm{D} \\
\text { Full analysis to predict the } \\
\text { frequency response of the } \\
\text { structure }\end{array}$ & $\begin{array}{l}\text { The reliability of the results } \\
\text { depends on the accuracy of the } \\
\text { calculation of the parameters of } \\
\text { the equivalent LC structure }\end{array}$ \\
\hline
\end{tabular}

More recently, a computationally efficient FSS analysis based on equivalent-circuit models was proposed (Costa, Monorchio \& Manara, 2012). The transmission and reflection properties of the FSS are evaluated through a first-order circuit approach based on computed lumped parameters of the most common FSS unit-cells. First, the L and C parameters representing a given FSS unit-cell are derived only one time at normal incidence and stored into a database. Secondly, the stored LC-equivalent circuits are perturbed to take into account the presence of arbitrarily thick dielectric slabs in the FSS structure, an arbitrary incident angle, or if a different periodicity with respect to the reference periodicity is adopted. The perturbed lumped parameters are then included in an equivalent transmission line for computing the response of generic frequency-selective-surface configurations with no additional computational effort.

Another efficient technique to derive the response charateristics of periodic arrays, made by very thin wire and arbitrary 
3-D type of elements is presented in (Mittra, Pelletti, Arya, Bianconi, McManus, Monorchio \& Tsitsas, 2012) and it is based on a generalised version of the waveguide simulator (WGS) approach. With this procedure it is possible to overcome the difficulty encountered with a Finite Method to compute the scattered field, because a reduction of the time-step in the simulation of the periodic structures is not demanded with an increased angle of incidence.

Another efficient technique to analyse a multi-layered non-resonant FSS comprising of metallic grids and patches, is proposed in (Jayaprakash\&Kavitha, 2014). With this approach it is not necessary to do a full 3-D EM analysis, because the structure is analyzed with an equivalent cascaded transmission line model. The equivalent circuit parameters (LC) estimated for the free standing metal layers based on a semi-analytical approach were found to differ from the analytical equations by a constant correction factor. The performance of the proposed cascaded transmission line model obtained using the estimated equivalent circuit parameters for the individual metal layers agreed very well with 3-D simulations. This method was shown to work for both single and multi-layered miniaturized FSS. Table I finally summarizes the most notable numerical techniques for the simulation of FSS structures in terms of pros and cons.

\section{Conclusions}

Significant research outcomes on FSS in the last 50 years have been here reviewed. A special focus is dedicated to the analysis and characterization of curved and finite-size structures. In fact, when periodicity is altered by truncation and/or non-zero curvature of the FSS, Floquet's theory cannot be rigorously applied and computationally heavy alternatives need to be carried out. Although this is a very common scenario, the literature dedicated to this particular aspect of FSS theory is sparse and lacks of a unified approach. This review paper groups together the most significant research paper on this topic in order to categorize valuable formulations and numerical solutions.

\section{Acknowledgements}

This work was supported by POR Campania FSE 2007 - 2013 "Materiali e Strutture Intelligenti" Rete di Eccellenza MASTRI CUP B25B09000010007.

\section{References}

Agrawal, V. D., \& Imbriale, W. A. (1979). Design of a dichroic Cassegrain subreflector. IEEE Transactions on Antennas and Propagation, 27(4), 466-473. http://dx.doi.org/10.1109/TAP.1979.1142119

Bosiljevac, M., \& Šipus, Z. (2008). Hybrid method for the analysis of curved electromagnetic structures. IEEE50th International Symposium ELMAR-2008, Zadar, Croatia, 613-616.

Braz, E. C., \& Campos, L. P. S. (2014). Dual/wide band multifractal frequency selective surface for applications in Sand X-band. Microwave \& Optical Tech. Letters, 56(10), 2217-2222. http://dx.doi.org/10.1002/mop.28561

Chambers, B., \& Tennant, A. (1994) Design of Wideband Jaumann Radar Absorbers with Optimum Oblique Incidence Performance. Electronics Letters, 30, 1530-1532. http://dx.doi.org/10.1049/el:19941023

Chen C., Zhu, Y., Zhao, Y., Lee, J. H., Wang, H., Bernussi, A., Holtz, M., \& Fan, Z. (2010). VO2 multidomain heteroepitaxial growth and terahertz transmission modulation. Applied Physics Letters, 97(21). http://dx.doi.org/10.1063/1.3519361

Costa, F., Monorchio, A., \& Manara, G. (2010). Analysis and Design of Ultra Thin Electromagnetic Absorbers Comprising Resistively Loaded High Impedance Surfaces. IEEE Transactions on Antennas and Propagation, 58, 1551-1558. http://dx.doi.org/10.1109/TAP.2010.2044329

Costa, F., Monorchio, A., \& Manara, G. (2012). Efficient Analysis of Frequency-Selective Surfaces by a Simple Equivalent-Circuit Model. IEEE Antennas and Propagation Magazine,54(4), 35-48. http://dx.doi.org/10.1109/TAP.2010.2044329

Cwik, T., \& Mittra, R. (1988). The Effects of the Truncation and Curvature of Periodic Surfaces: A Strip Grating. IEEE Transactions on Antennas and Propagation,36(5), 612-622. http://dx.doi.org/10.1109/8.192137

Dick, M. J. (1981). An ellipsoidal frequency selective surface. Proceedings of Antenna Appl. Symp., Urbana-Champaign, IL.

Floquet, M. G. (1883). Sur les equations différentielles linéaires a coefficients périodiques. Annale Ecole Normale Superieur, 2(12), 47-88.http://www.numdam.org/item?id=ASENS_1883_2_12_47_0.

Genovesi, S., Costa, F., \& Monorchio, A. (2012) Low-Pro file Array With Reduced Radar Cross Section by Using Hybrid Frequency Selective Surfaces. IEEE Transactions on Antennas and Propagation, 60(5), 2327-2335. http://dx.doi.org/10.1109/TAP.2012.2189701

Glisson, A. W., \& Wilton, D. R. (1980). Simple and efficient numerical methods for problems of electromagnetic radiation and scattering from surfaces. IEEE Transactions on Antennas and Propagation, 28(5), 593-603. 
http://dx.doi.org/10.1109/TAP.1982.1142818

Harrington, R. F. (1993). Field computation by moment methods. Wiley-IEEE Press, New York. http://dx.doi.org/10.1109/9780470544631

Hu, X.-D., Zhou, X.-L., Wu, L.-S., Zhou, L., \& Yin, W.-Y. (2009). A miniaturized dual-band frequency selective surface (FSS) with closed loop and its complementary pattern. IEEE Antennas Wireless Propagation Letters, 18, 1374-1377. http://dx.doi.org/10.1109/LAWP.2009.2039110

Jayaprakash, P., \& Arunachalam, K. (2014). Semi Analytical Model for Non-Resonant Layered Frequency Selective Surfaces (FSS). Proceedings of the Progress In Electromagnetics Research Symposium, Guangzhou, China, 1767-1771.

Kastner, R., \& Mittra, R. (1984).A combination of the spectral-iterative technique and the conjugate gradient method for analyzing finite-sized frequency selective surfaces with rectangular patches. Proceedings of IEEE Antennas Propagat. Soc. Int. Symp. Dig., Boston, MA, 925-928. http://dx.doi.org/10.1109/LAWP.2009.2039110

Knott E. F. (1985). A progression of high-frequency RCS prediction techniques. Proceedings IEEE, 73(2), 252-264. http://dx.doi.org/10.1109/PROC.1985.13137

Kornbau, T. W. (1984). Analysis of Periodic Arrays of Rotated Linear Dipoles, Rotated Crossed Dipoles, and of Biplanar Dipole Arrays in Dielectric. Ph.D. Dissertation, Ohio State Univ., Dept. of Electrical Eng., Columbus. https://etd.ohiolink.edu/rws_etd/document/get/osu1383564568/inline

Kouyoumjian R. G. (1965). Asymptotic high-frequency methods. Proceedings IEEE, 53(8), 864-876. http://dx.doi.org/10.1109/PROC.1965.4065

Kron G. (1953). A set of principles to interconnect the solutions of physical systems. Journal of Applied Physics, 24(8), 965-980. http://dx.doi.org/10.1063/1.1721447

Laza, V. A., Matekovits, L., \& Vecchi, G. (2005). Synthetic-functions decomposition of large complex structures. IEEE Antennas and Propagation Society International Symposium, Albuquerque, 3925-3928. http://dx.doi.org/10.1109/APS.2006.1711483

Li, M. K., \& Chew, W. C. (2007). Wave-field interaction with complex structures using equivalence principle algorithm. IEEE Transactions on Antennas and Propagation, 55(1), 130-138. http://dx.doi.org/10.1109/TAP.2006.888453

Li, W., Zhang, T., Yang, G., \& Wu, Q. (2012). A Novel Frequency Selective Surface with Improved Miniaturization Performance. Journal of Electromagnetic Analysis and Applications, 4, 108-111. http://dx.doi.org/10.4236/jemaa.2012.43014

Matekovits, L., Vecchi, G., Dassano, D., \& Orefice, M. (2001). Synthetic function analysis of large printed structures: the solution space sampling approach. IEEE Antennas and Propagation Society International Symposium, Boston, 2, 568-571. http://dx.doi.org/10.1109/APS.2001.959787

Meng, Z. J., Lu, M. Y., Wu, Z., \& Huang, J. (2009).One-Dimensional Curved Effects of Finite Slot Arrays. APMC Asia Pacific Microwave Conference, Singapore, 9-12. http://dx.doi.org/10.1109/APMC.2009.5385346

Mittra, R., Pelletti, C., Arya, R. K., Bianconi, G., McManus, T., Monorchio, A., \& Tsitsas, N. (2012). New numerical techniques for efficient and accurate analysis of FSSs, EBGs and Metamaterials. Proceedings of the 6th European Conference on Antennas and Propagation (EUCAP), 2669-2670. http://dx.doi.org/10.1109/EuCAP.2012.6206506

Munk, B. A. (2000). Frequency Selective Surfaces: Theory and Design. John Wiley \& Sons Inc, New York. http://dx.doi.org/10.1002/0471723770

Munk, B. A., Munk, P., \& Pryor, J. (2007). On Designing Jaumann and Circuit Analog Absorbers (CA Absorbers) for Oblique Angle of Incidence. IEEE Transactions on Antennas and Propagation, 55, 186-193. http://dx.doi.org/10.1109/TAP.2006.888395

O’Nians, F., \& Matson, J. (1966). Antenna feed system utilizing polarization independent frequency selective intermediate reflector. U.S. Patent 3231892.

Pelletti, C., Bianconi, G., Mittra, R., \& Monorchio, A. (2013). Efficient modeling of conformal Frequency Selective Surfaces using the characteristic basis function method. Proceedings of the International Symposium on Electromagnetic Theory, $704-707$.

Pelletti, C., Bianconi, G., Mittra, R., \& Monorchio, A. (2013b). Analysis of Finite Conformal Frequency Selective Surfaces via the Characteristic Basis Function Method and Spectral Rotation Approaches. IEEE Antennas and Wireless Propagation Letters, 12, 1404-1407. http://dx.doi.org/10.1109/LAWP.2013.2287377 
Philips, B., Parker, E. A., \& Langley, R. J. (1995). Ray tracing analysis of the transmission performance of curved FSS. IEE Proc. of Microwaves, Antennas \& Prop, 193-200. http://dx.doi.org/10.1049/ip-map:19951896

Prakash, V. V. S., \& Mittra, R. (2003). Characteristic basis function method: a new technique for efficient solution of method of moments matrix equation. Microw Opt TechnolLett,36(2), 95-100. http://dx.doi.org/10.1002/mop.11085

Rao S. M., Wilton D. R., \& Glisson A. W. (1982).Electromagnetic scattering by surfaces of arbitrary shape. IEEE Transactions on Antennas and Propagation, 30(3), 409-412. http://dx.doi.org/10.1109/TAP.1982.1142818

Ren L., Pint, C. L., Arikawa, T., Takeya, K., Kawayama, I., Tonouchi, M., Hauge, R. H., \& Kono, J. (2012). Broadband Terahertz Polarizers with Ideal Performance Based on Aligned Carbon Nanotube Stacks. Nano Letters, 12 (2), 787790. http://dx.doi.org/10.1021/nl203783q

Ruvio, G., \& Leone, G. (2014). State-of-the-art of Metamaterials: Characterization, Realization and Applications. Studies in Engineering and Technology,1(2), 38-47. http://dx.doi.org/10.11114/set.v1i2.456

Schneider, S. W., \&Munk, B. A. (1994).The scattering properties of "Super Dense" arrays of dipoles.IEEE Transactions on Antennas and Propagation, 42(4), 463-472. http://dx.doi.org/10.1109/8.286213

Seman, F. C., Cahill, R., \& Fusco, V. (2009).Low Profile Salisbury Screen Radar Absorber with High Impedance Ground Plane. Electronics Letters, 45, 10-12. http://dx.doi.org/10.1049/el:20093098

Semyoung, O., Hanjun, L., Joo-Ho, J., \& Gil-Young, L. (2014). A Novel Wideband Miniaturized-Element Frequency Selective Surface. International Journal of Microwave Science and Technology, Article ID 857582.http://dx.doi.org/10.1155/2014/857582

Šipus, Z., Burum, N., Skokic, S., \& Kildal, P. S. (2006). Analysis of spherical arrays of microstrip antennas using moment method in spectral domain. IEE Proc. Microw. Antennas Propag, 153(6), 533-543. http://dx.doi.org/10.1049/ip-map:20050262

Šipus, Z., Lanne, M., \& Josefsson, L. (2006). Moment method analysis of circular cylindrical array of waveguide elements covered with a multilayer radome. IEE Proc. Microw. Antennas Propag, 153(1), $29-37$. http://dx.doi.org/10.1049/ip-map:20045067

Stupfel, B., \& Pion, Y. (2005). Impedance boundary conditions for finite planar and curved frequency selective surfaces. IEEE Transactions on Antennas and Propagation, 53(4), 1415-1425. http://dx.doi.org/10.1109/TAP.2005.844417

Ulrich, R. (1967). Far-infrared properties of metallic mesh and its complementary structure. Infrared Phys. 7, 37-55. http://dx.doi.org/10.1016/0020-0891(67)90028-0

van de Water, A. M., de Hon, B. P., van Beurden, M. C., Tijhuis, A. G., \& de Maagt, P. (2005) Linear embedding via Green's operators: a modeling technique for finite electromagnetic band-gap structures. Phy. Rev. E, 72(5), 1-11. http://dx.doi.org/10.1103/PhysRevE.72.056704

Vegesna, S., Zhu, Y., Bernussi, A., \& Saed, M. (2012). Terahertz Two-Layer Frequency Selective Surfaces With Improved Transmission Characteristics. IEEE Transactions on Terahertz Science and Technology, 2(4), 441-448. http://dx.doi.org/10.1109/TTHZ.2012.2202035

Vegesnaa, S., Zhuab, Y., Zhaoab, Y., Fanab, Z., Bernussiab, A., \& Saeda, M. (2014). Terahertz frequency selective surface with reconfigurable polarization characteristics using vanadium dioxide. Journal of Electromagnetic Waves and Applications, 28(1), 83-90. http://dx.doi.org/10.1080/09205071.2013.857278

Wu, R., Zhang, H., Xu, Z., Yang, Z., \& Lin, Y. (2014). A Novel Miniaturized Frequency Selective Surface with Stable Performances. Progress In Electromagnetics Research Symposium Proceedings, Guangzhou, China 1647-1650.

Wu, T. K. (1995). Frequency Selective Surface and Grid Array. John Wiley \& Sons Inc, New York.

Yang, H. Y., Gong, S. X., Zhang, P. F., Zha, F. T., \& Ling, J. (2009). A novel miniaturized frequency selective surface with excellent center frequency stability. Microwave Optical Technology Letters, 51, 2513-2516. http://dx.doi.org/10.1002/mop.24604

Yu, C., \& Lu, C. C. (2005). Analysis of curved frequency-selective surfaces using the hybrid volume-surface integral equation approach. Microwave Optical Technology Letters, 45(2), 107-112. http://dx.doi.org/10.1002/mop.20738

Yu, F., Qu, S., Wang, J., Huang, H., \& Wang, J. (2014).The Design of Band-pass Frequency Selective Surface with All Dielectric Metamaterial. Progress In Electromagnetics Research Symposium Proceedings, Guangzhou, China, 1878-1880. 
Yu, F., Qu, S., Xu, Z., \& Wang, J. (2011). Investigations on the design of all-dielectric frequency selective surfaces. Progress In Electromagnetics Research Symposium Proceedings, Suzhou, China, 472-475.

Zhao, Y., Lee, J. H., Zhu, Y., Nazari, M., Chen, C., Wang, H., Bernussi, A., Holtz, M., \& Fan, H. (2012). Structural, electrical, and terahertz transmission properties of $\mathrm{VO}_{2}$ thin films grown on c-, $\mathrm{r}-$, and m-plane sapphire substrates. Journal of Applied Physics, 111(5). http://dx.doi.org/10.1063/1.3692391

\section{(c) $\mathrm{EY}$}

This work is licensed under a Creative Commons Attribution 3.0 License. 